\title{
EFECTIVIDAD Y EFECTOS SECUNDARIOS DEL TRATAMIENTO CON CANABINOIDES EN DOLOR NEUROPÁTICO DE TIPO CENTRAL EN PACIENTES CON ESCLEROSIS MÚLTIPLE
}

\author{
Janice Maldonado M.D. ${ }^{1}$, Andrés Mauricio Álvarez Pinzón M.D. ${ }^{2}$, Mayerli Rodríguez Martínez M.D. ${ }^{3}$ \\ ${ }^{1}$ División de Neurología Clínica, Universidad de Miami, Escuela de Medicina "Leonard Miller", Miami (FL), USA \\ ${ }^{2}$ Universidad El Bosque, Bogotá, Colombia. ${ }^{3}$ Universidad Militar Nueva Granada, Bogotá, Colombia.
}

\begin{abstract}
Resumen
La Esclerosis Múltiple (EM) es una enfermedad neurodesmielinizante de origen inmunológico que afecta a personas entre los 16 a los 35 años de edad y en la cual del $36 \%$ al $82 \%$ de los pacientes manifiestan dolor como síntoma común. La EM tiene una prevalencia mundial de 4,41 por cada 100.000 habitantes, afectando alrededor de 2,5 millones de personas en todo el mundo y cerca de 400.000 en Estados Unidos; en Colombia es de 4,3 por cada 100.000 habitantes, presentándose la mayoría de los casos en Risaralda seguido por Bogotá. Los síndromes de dolor neuropático de tipo central se presentan por lesión o disfunción del sistema nervioso central, causando discapacidad y deterioro de la calidad de vida de los pacientes. En este artículo se hace una revisión sobre la efectividad analgésica y efectos secundarios de los canabinoides en el tratamiento de dolor neuropático de tipo central en pacientes con EM. Se realizó una estrategia de búsqueda identificando ensayos clínicos aleatorizados sobre tratamientos de dolor neuropático de tipo central en EM con canabinoides y se seleccionaron seis estudios que compararon canabinoides vs placebo. Basados en los artículos revisados se puede concluir, que hay una disminución significativa del dolor neuropático de tipo central en pacientes que padecen EM con la administración de canabinoides y los efectos secundarios son leves, siendo los más frecuentes vértigo, xerostomía, somnolencia y alteraciones en el equilibrio los cuales no alteran la calidad de vida del paciente.
\end{abstract}

Palabras clave: esclerosis múltiple, dolor, clínicas del dolor, canabinoides, dronabinol

\section{EFFECTIVITY AND SIDE EFFECTS OF CENTRAL NEUROPATHIC PAIN TREATMENT WITH CANNABINOIDS IN PATIENTS WITH MULTIPLE SCLEROSIS}

\begin{abstract}
Multiple Sclerosis (MS) is an immunological neuro-demyelinating disease that affects population between 16 to 35 years. Pain is the most common symptom with $36 \%$ to $82 \%$ of patients reporting pain. MS has a worldwide prevalence of 4.41 per 100,000 people, affecting about 2.5 million people around the world and 400,000 in the United States; in Colombia 4.3 per 100,000 inhabitants suffer from this disease where most of the cases have been reported in the region of Risaralda and the capital city Bogotá. Central neuropathic pain syndrome is due to injury and dysfunction of the central nervous system, causing disability and impairing quality of life of patients. In this article, a review of the analgesic effectiveness and side effects of cannabinoids in the treatment of central neuropathic pain in
\end{abstract}

* Correspondencia: Mauricio Álvarez mauricioalvarez07@yahoo.com. Dirección correspondencia: 9000 Royal Palm Blvd E604 Coral Springs, FL 33065.

Recibido: Marzo 4 de 2010. Aceptado: Junio 4 de 2010. 
patients with MS is conducted. A search strategy was performed to identify randomized clinical trials on the treatment of central neuropathic pain in MS with cannabinoids; six selected studies comparing cannabinoids versus placebo were selected and after analyzing them, it was found a significant decrease in pain with dronabinol (cannabinoid) treatment and low side effects. The most common side effects were drowsiness, dry mouth, dizziness and impaired balance, which did not compromise the quality of life of patients. Based on the review of articles it is concluded that there is a significant reduction in central neuropathic pain in patients with MS in therapy with cannabinoids and that side effects of treatment are very low.

Keywords: multiple sclerosis, pain, pain clinics, adverse effects, cannabinoids, dronabinol

\title{
EFICÁCIA E EFEITOS SECUNDÁRIOS DO TRATAMENTO COM CANABINÓIDES EM DOR NEUROPÁTICA TIPO CENTRAL EN PACIENTES CON ESCLEROSE MULTIPLA
}

\begin{abstract}
Resumo
Esclerose Múltipla (EM) é uma doença neurodesmielinizante de origem imune que afeta pessoas entre 16 e 35 anos, onde $36 \%$ a $82 \%$ dos pacientes relatam dor como um sintoma comum. MS tem uma prevalência mundial de 4,41 por 100.000 pessoas, afetando cerca de 2,5 milhões de pessoas no mundo e cerca de 400.000 nos Estados Unidos; na Colômbia é de 4,3 por 100.000 habitantes, apresentando-se a maioria dos casos em Risaralda seguida por Bogotá. Síndromes de dor neuropática do tipo central são apresentadas por uma lesão ou disfunção do sistema nervoso central, causando incapacidade e redução da qualidade de vida dos pacientes. Neste trabalho, uma análise da eficácia analgésica e efeitos colaterais dos canabinóides no tratamento do tipo de dor neuropática central em pacientes com EM. Realizamos uma estratégia de pesquisa para identificar estudos clínicos randomizados sobre o tratamento da dor neuropática em MS tipo central com canabinóides, furam seis estudos selecionados comparando canabinóides versus placebo. Com base nos artigos concluem que há uma redução significativa da dor neuropática da tipo central em pacientes com EM com a administração de canabinóides e os efeitos colaterais são leves, os mais comuns são tontura, sonolência, xerostomia e distúrbios do equilîbrio que não alterou a qualidade de vida dos pacientes.
\end{abstract}

Palavras-chave: esclerose múltipla, dor, clínicas de dor, canabinóides, dronabinol

\section{Introducción}

La Esclerosis Múltiple (EM) fue definida inicialmente por Robert Carswell en 1983 como la representación de "una notable lesión en la médula espinal acompañada de atrofia", la cual fue una descripción aproximada de la anatomía patológica y características clínicas de esta enfermedad del Sistema Nervioso Central (SNC) (1). La EM es una enfermedad que afecta alrededor de 2,5 millones de personas a nivel mundial, con una prevalencia de 4,41 por 100.000, cerca de 400.000 en Estados Unidos (2,3). En Colombia hay muy pocos estudios respecto a su epidemiología, pero con los realizados durante el período comprendido entre julio de 1995 y junio de 2000 se determinó que los departamentos en los que la patología es más frecuente son Antioquia, Caldas, Santander, Risaralda y Bolívar, con una prevalencia (casos de EM por 100.000 habitantes) que varía entre 1,48 en Antioquia (IC95\% $1,12 ; 1,78)$ y 4,98 en Risaralda (IC95\% 3,52; 6,43) y se identificó que el $72 \%$ de los individuos con EM son del sexo femenino (3-5). En Bogotá se encontró que la prevalencia promedio es de 4,41 por 100.000 habitantes, en hombres es de 2,71/100.000 y en mujeres de $5,98 / 100.000$ con una diferencia de $\mathrm{p}<$ de 0,001 (5).

En la última década los canabinoides han sido muy estudiados en el tratamiento de este tipo de dolor; sin 
embargo, no se han encontraron ensayos clínicos que muestren una comparación entre los diferentes estudios existentes en el tratamiento del dolor neuropático de tipo central con canabinoides en EM, que permitan concluir cual es el medicamento más efectivo para el control del dolor en los pacientes con esta patología específica y que produzca menos efectos secundarios; es por ello que se decidió realizar esta revisión sobre la efectividad y seguridad de los canabinoides para el tratamiento del dolor neuropático en pacientes con EM. Es importante tener en cuenta que las estrategias en el cuidado de estos pacientes son multifacéticas e involucran un diagnóstico precoz, tratamiento adecuado, educación al paciente, del núcleo familiar y el suministro de un sistema de salud de apoyo para mejorar la calidad de vida con un tratamiento integral en un enfoque biosicosocial.

\section{Fisiopatogenia de la EM}

La patogénesis de la EM se basa en el factor genético con predominio de los alelos del Complejo Mayor de Histocompatibilidad (CMH) II, los marcadores DR15, DQ6, DRB1 y DR4 entre otros; y el factor ambiental (6-8). Desde el punto de vista inmunológico la falta de mecanismos de regulación local dentro del cerebro en los sitios de inflamación, hace que predominen los infiltrados perivasculares de células $\mathrm{CD} 8+$, provocando formación de placas que se agrupan en torno a los ventrículos laterales, el cuerpo calloso, la corteza cerebral, la materia blanca subcortical, los nervios ópticos y el tronco cerebral. El edema secundario a la reacción inflamatoria es producido por los linfocitos T que segregan interleucina 17 (IL-17) y el control dado por la IL-23. IL-17 e IL-22 afectan la barrera hematoencefálica, permitiendo la penetración eficaz de las células T ayudadoras 17 (Th 17) en el cerebro, donde generan una reacción inflamatoria a nivel neural produciendo efectos desmielinizantes y la inducción de apoptosis por cascada de señalización (6-8).

En el inicio de la etapa secundaria progresiva, las zonas ya existentes con desmielinización axonal difusa y degeneración neuronal, son lesiones permanentes por acumulación de procesos de fosforilación, y crecen lentamente por la expansión de la inflamación cerebral; van desvaneciendo la sustancia blanca y la activación difusa del parénquima microglial lo que genera anomalías en la sustancia blanca que no se evidencian en la resonancia magnética (9-11). El curso clínico se caracteriza por ser progresivo y degenerativo donde los principales síntomas son: alteración de la sensibilidad, espasticidad de los miembros, disminución de la fuerza motora, disartria, dipoplía, disminución de la agudeza visual y el bien reconocido dolor neuropático, el cual surge como consecuencia directa de una lesión o enfermedad que afecta a nivel central el sistema somato sensorial (12).

\section{Dolor Neuropático Central}

La Organización Internacional de la Asociación para el Estudio del Dolor (IASP) lo define como "dolor iniciado o causado por una lesión primaria o disfunción del SNC". Recientemente, otra definición se ha introducido y la cual sugiere que el dolor central es "el dolor que surge como consecuencia directa de una lesión o enfermedad que afecta a nivel central el sistema somato sensorial" (13-15).

A raíz de esta nueva propuesta, para poder definir el dolor como neuropático éste debe tener una distribución neuroanatómica, con historia de una lesión o enfermedad que afecte el sistema el SNC y con un diagnóstico que pueda explicar su presencia (14). La identificación del dolor neuropático es importante y requiere de varias pruebas para excluir un dolor periférico de tipo nociceptivo. Al evaluar el dolor los métodos utilizados deben tener varias herramientas de selección que permitan diferenciar entre el neuropático y el no neuropático (15).

Las diferentes formas clásicas de presentación del dolor son: dolor extenso de causa inexplicable, evidencia de déficit sensorial, dolor tipo ardor o corriente, dolor que se presenta con un ligero rose en la piel y crisis de dolor independientes de estímulo. El dolor es un síntoma frecuente y molesto asociado con EM; varios estudios realizados sugieren que la prevalencia de dolor es diferente en mujeres que en hombres, presentándose más frecuente en la población femenina; en Estados Unidos la incidencia de dolor relacionado con la EM varía de $53 \%$ a $86 \%$ de los pacientes (16-19).

La falta de criterios claros para el diagnóstico del dolor neuropático hace que este sea difícil. La escala de dolor neuropático (EDN) es la única herramienta que actualmente ha sido validada para la evaluación del dolor y ha sido desarrollada para valorar las diferentes cualidades asociadas con el. El uso de esta escala ha permitido la disminución de sesgos, ya que es sensible a la medición de resultados de intervenciones terapéu- 
ticas y a que el dolor es un síntoma dependiente de paciente $(20,21)$.

Para el manejo del dolor neuropático en EM existen diversos medicamentos entre ellos están antidepresivos tricíclicos, anticonvulsivantes, opiodes y cannabinoides entre otros. Dentro de los canabinoides el más estudiado ha sido el dronabinol (22-25). En varios estudios en los que se utilizaron canabinoides se observó una adecuada tolerancia a ellos y los efectos secundarios que se presentaron fueron somnolencia, xerostomía, vértigo, y alteración del equilibrio que no limitaban las actividades diarias de los pacientes. La escala empleada en estos estudios para evaluar la disminución del dolor neuropático fue la escala análoga del dolor cuyos valores están entre 0 a 10 , en donde 10 es un dolor no tolerable e incapacitante que limita la vida diaria y 0 es la ausencia de dolor.

\section{Estudios revisados}

Para hacer esta revisión se tomaron los artículos publicados entre Enero 2000 y Enero 2010 en revistas indexadas, sobre estudios que estuvieran relacionados con el uso de canabinoides para el tratamiento del dolor neuropático de tipo central en pacientes con EM, en pacientes de cualquier sexo, los cuales evaluaran la intensidad del dolor antes y después del tratamiento utilizando la escala numérica de dolor, y los resultados de la evaluación metodológica de los artículos por medio de la escala de Jadad fuesen mayores o iguales a tres. Todos los artículos debían ser sobre ensayos clínicos aleatorizados, controlados con placebo.

Después de hacer una extensiva revisión en las bases de datos PubMed (1965 - Enero 2010), Cochrane (2000 - Enero 2010), Lylacs (2000 - Enero 2010), Proquest (2000 - Enero 2010), Medline (1965 - Enero de 2010), se encontraron 48 artículos en revistas indexadas, de los cuales se excluyeron 32 por ser de diseños diferentes a ensayos clínicos controlados (revisiones sistemáticas, artículos descriptivos, comentarios); de los 16 artículos escogidos se excluyeron aquellos que no mostraban tratamiento para el manejo del dolor neuropático central en EM, sino que se referían al tratamiento del dolor neuropático en general. Como resultado final se encontraron seis artículos que cumplieron todos los criterios de selección establecidos para ésta revisión (Figuras 1 y 2 ).

En los diferentes estudios se compararon diferentes canabinoides contra placebo y se observó que el dronabinol oral en una dosis máxima de $10 \mathrm{mg} /$ día reduce
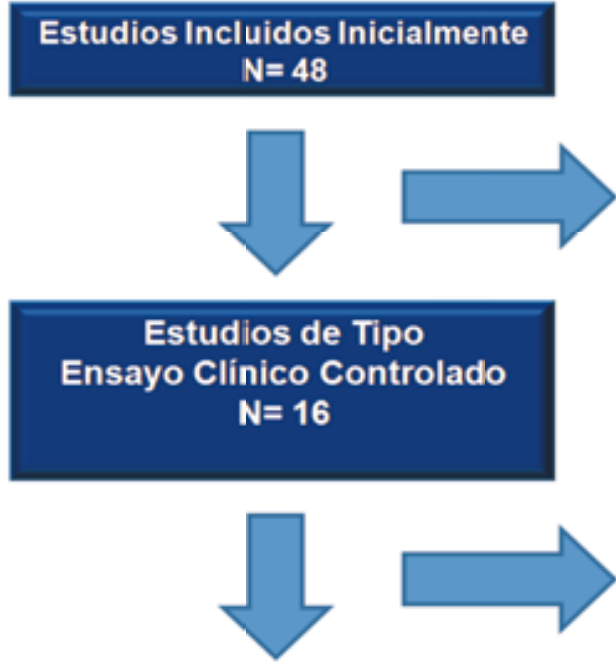

\section{Estudios Seleccionados para} Revisión Sistematica $\mathrm{N}=6$

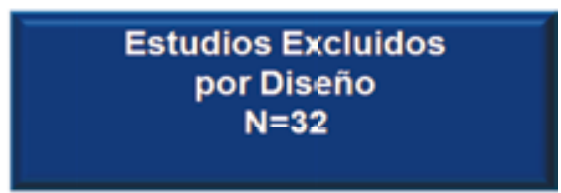

Estudios Excluidos por no Cumplir Criterios de Inclusión $\mathrm{N}=10$

FIGURA 1. Proceso de búsqueda y selección de estudios. 


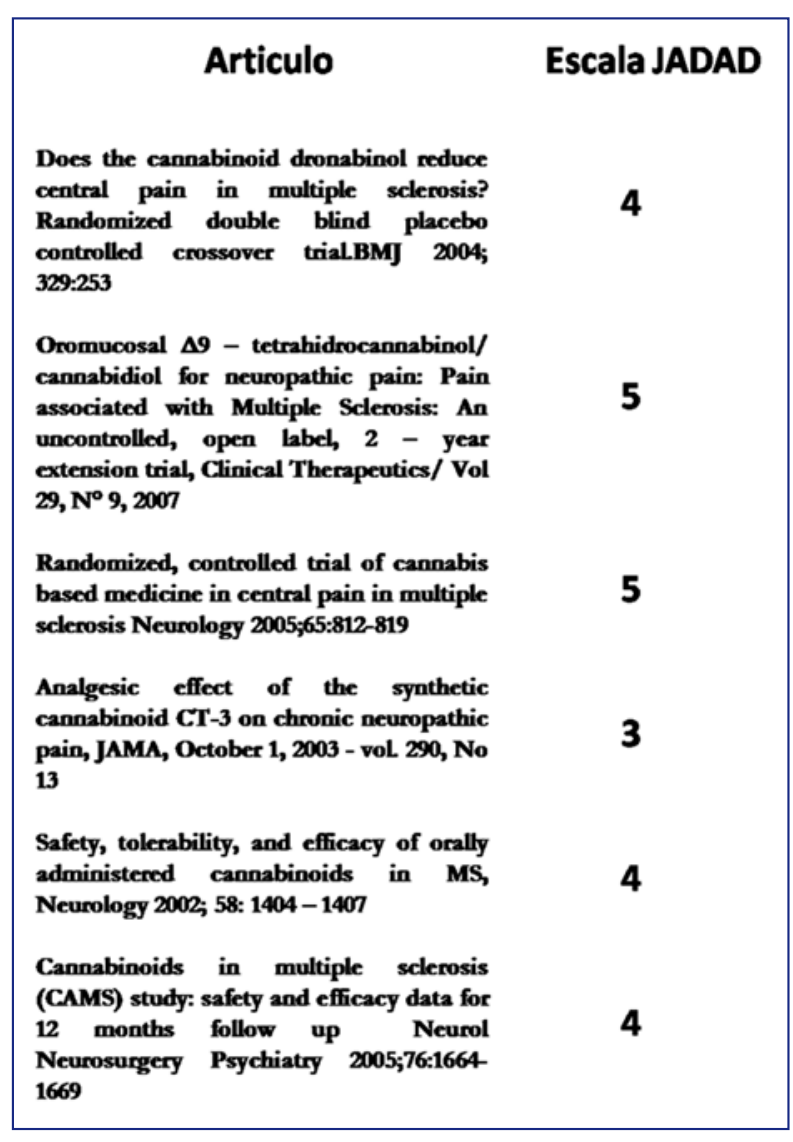

FIGURA 2. Evaluación de los artículos referenciados según escala de JADAD.

significativamente el dolor neuropático central en los pacientes (de tres puntos en pacientes que referían un dolor inicial de tres a diez puntos), al compararlo con los resultados obtenidos de los que se les había suministrado placebo y los efectos secundarios producidos eran tolerables (Figura 3). La reducción primaria, incluyendo la intensidad, irradiación y progresión del dolor en comparación al placebo fue de un $31 \%$ (Figura 3) $(26,27)$.

La escala para evaluar la disminución del dolor neuropático en la esclerosis múltiple fue la escala análoga del dolor de cero a diez donde diez es un dolor no tolerable, incapacitante que limita la vida diaria y cero es la ausencia de dolor. Se observó en los diferentes estudios una disminución significativa del dolor en comparación con el placebo.

La cantidad de efectos adversos y tolerabilidad depende de la tolerancia al medicamento de cada uno de los pacientes y es proporcional a la cantidad de días de administración; en los estudios publicados en los artículos revisados se observó una buena tolerancia y pocos efectos adversos durante la administración del canabinoides. Los observados fueron somnolencia, xerostomía, y vértigo que no comprometieron la calidad de vida. Los efectos secundarios disminuyeron significativamente tras su administración por un período mayor de cuatro semanas (Figura 3).

Hacen falta estudios específicos y comparativos en el tratamiento del dolor neuropático central en EM que permitan realizar un análisis específico para la consejería en la práctica clínica. Los ensayos clínicos actualmente disponibles sólo permiten comparar la efectividad y los efectos secundarios del canabinoides con el placebo porque no hay estudios comparativos directos que determinen su efectividad y seguridad frente a otros medicamentos.

Después de hacer la revisión y basados en los artículos publicados se puede concluir que los canabinoides se asocian con una disminución clínica significativa de dolor neuropático en EM al ser comparado con el placebo. Los efectos adversos observados son vértigo, somnolencia y xerostomía, síntomas que no comprometieron la calidad de vida de los pacientes y disminuyeron notablemente tras su administración mayor a cuatro semanas. Los canabinoides a lo largo de la última década han sido una opción terapéutica en el tratamiento del dolor neuropático central en pacientes con esclerosis múltiple especialmente en Canadá y algunos estados de Norte América.

\section{Recomendaciones}

Es necesario realizar más ensayos clínicos específicos en el tratamiento del dolor neuropático central en EM, que comparen específicamente las diferentes opciones terapéuticas. Se debe tener en cuenta los canabinoides como una opción en el tratamiento del dolor neuropático en EM y realizar estudios comparativos de las diferentes opciones terapéuticas farmacológicas como anticonvulsivantes, canabinoides, opioides y antidepresivos tricíclicos, con tamaños de muestra adecuados, resultados estadísticamente significativos, clínicamente pertinentes, analizando los efectos secundarios presentados por la administración de cada medicamento y la intensidad del dolor utilizando la escala de evaluación del dolor neuropático en EM para valorar objetivamente su eficacia. 


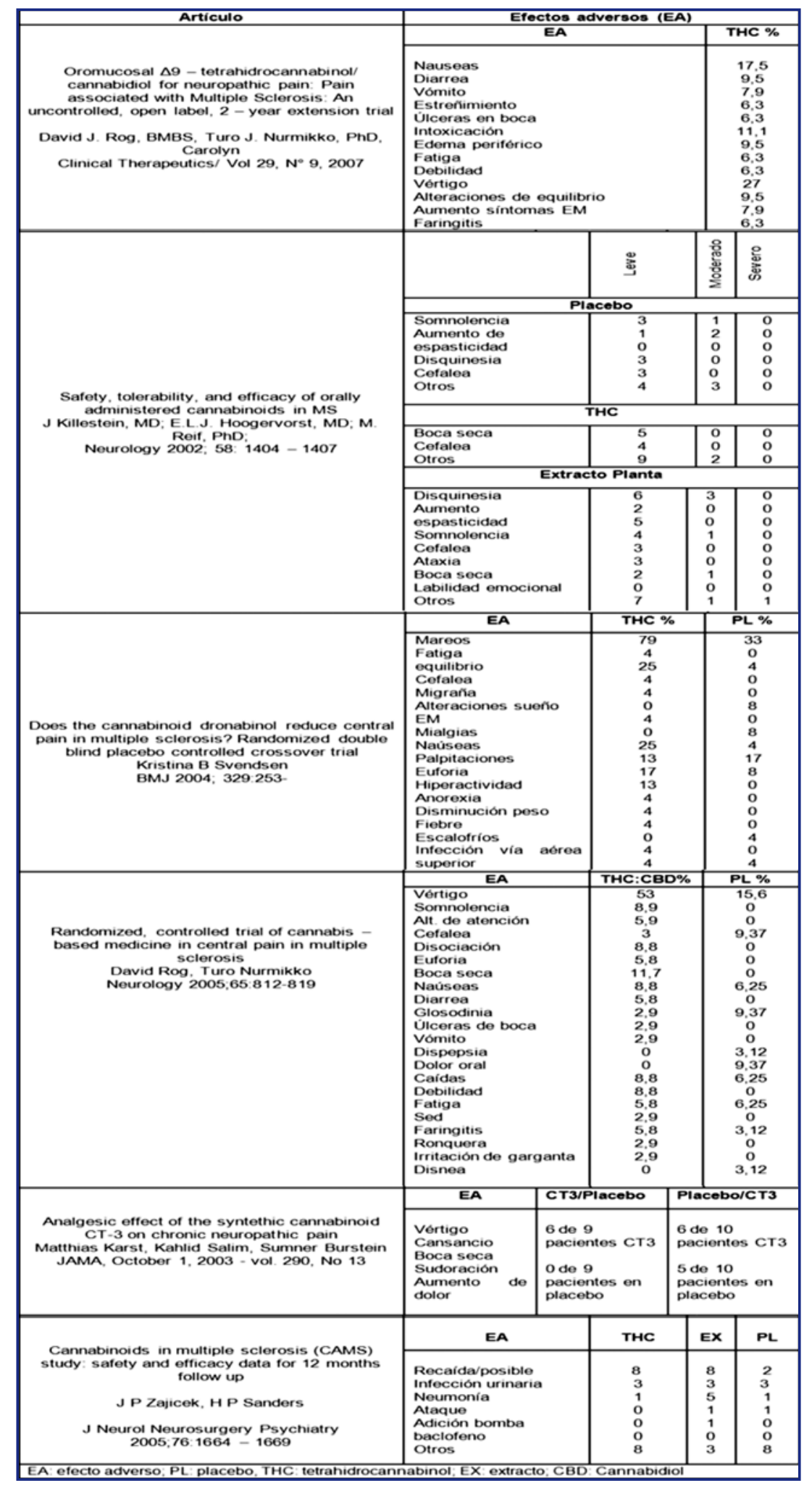

FIGURA 3. Efectos adversos de medicamentos empleados en los estudios científicos. 


\section{Referencias}

1. Stanley Finger. History of Neurology: A Happy State of Mind: A History of Mild Elation, Denial of Disability, Optimism, and Laughing in Multiple Sclerosis Arch Neurol 1998; 55: 241 250.

2. Korsakova S S. Multiple Sclerosis. Multiple Sclerosis. 2007;4:50-56.

3. Kurtzke J F. Una Nueva Evaluación de la Distribución de la Esclerosis Múltiple, Parte I. Acta Neurologica Scandinavica. 1975;51:110-136.

4. Sánchez J L, Aguirre C, Arcos-Burgos M, Jiménez-Ramírez I, Jiménez M E, Leon-Sarmiento F E, Pareja-Ángel J, PradillaArdila G, Uribe B, Uribe B, Villa-López A, Volcy-Gómez M, Palacio L G. Prevalencia de la Esclerosis Múltiple en Colombia. Revista Neurologica. 2000;31:1101-1103

5. Toro J, Sarmiento O L, Diaz del Castillo A, Satizabal C L, Ramírez J D, Montenegro A C, Góngora M C, Quiñones J A, Díaz A, Tobón A. Prevalence of Multiple Sclerosis in Bogotá, Colombia. Neuroepidemiology. 2007;28:33-38.

6. Peresedova A V, Baidina E V, Trifonova O V, Korepina O S, Gnezditskii V V, Krotenkova M V, Konovalov R N, Chernikova L A, Alekseeva N S, Kirichenko I M, Rebrova O Y, Zavalishin I A. Pathophysiological Aspects of the Formation of Neurological Deficit in Multiple Sclerosis. Neuroscience and Behavioral Physiology. 2009;39:110.

7. Compston A, Coles A. Multiple Sclerosis. Lancet. 2008;372:1502-17.

8. Confavreux C, Vukusic S. Natural History of Multiple Sclerosis: A Unifying Concept. Brain. 2006;129:606-616.

9. Fong J S, Rae-Grant A, Huang D. Neurodegeneration and Neuroprotective Agents in Multiple Sclerosis. Recent Patents on CNS Drug Discovery. 2008;3:153-65.

10. Kutzelnigg A, Lucchinetti C F, Stadelmann C. Cortical Demyelization and Diffuse White Matter Injury in Multiple Sclerosis. Brain. 2005;128:2705-2712.

11. Kuhle J, Pohl C, Mehling M. Lack of Association between Antimyelin Antibodies and Progression to Multiple Sclerosis. New England Journal of Medicine. 2007;356:371-378.

12. Bruce A C. Identification, Causation, Alleviation, and Prevention of Complications (ICAP) an Approach to Symptom and Disability Management in Multiple Sclerosis. Neurology. 2008;71(3):14-20.

13. Merskey H, Bogduk N. Classification of Chronic Pain: Descriptions of Chronic Pain Syndromes and Definitions of Pain Terms. Seattle. 1994

14. Treede R D, Jensen T S, Campbell J N. Neuropathic Pain: Redefinition and a Grading System for Clinical and Research Purposes. Neurology. 2008;70:1630-1635.
15. Galer B S, Jensen M P. Development and Preliminary Validation of a Pain Measure Specific to Neuropathic Pain: The Neuropathic Pain Scale. Neurology. 1997;48:332-338.

16. Finnerup N B. A Review of Central Neuropathic Pain States. Current Opinion in Anesthesiology. 2008;21:586-9.

17. Österberg A, Boivie J, Thomas K A. Central Pain in Multiple Sclerosis- Prevalence and Clinical Characteristics. European Journal of Pain. 2005;9:531-542.

18. Newland P. Pain in Momen with Relapsing-remitting Multiple Sclerosis and in Healthy Women: A Comparative Study. Journal of Neuroscience Nursing. 2008;40:262-8.

19. Grasso M G. Pain in Multiple Sclerosis: A Clinical and Instrumental Approach. Multiple Sclerosis. 2008;14:506-513.

20. Rog D J, Nurmikko T J, Friede T, Young C A. Validation and Reliability of the Neuropathic Pain Scale (NPS) in Multiple Sclerosis. The Clinical Journal of Pain. 2007;23:473-481.

21. Solaro C, Brichetto G, Amato M P, Cocco E, Colombo B, D'Aleo G, Gasperini C, Ghezzi A, Martinelli V, Milanese C, Patti F, Trojano M. The Prevalence of Pain in Multiple Sclerosis: A Multicenter Cross-sectional Study. Neurology. 2004;63:919-921.

22. Ross A P, APN, MSN, CNRN, MSCN. Strategies for Optimal Disease Management, Adherence and Outcomes in Multiple Sclerosis Patient. Neurology. 2008;71:1-2.

23. Rodríguez U, Carrillo E, Soto E. Canabinoides: Neurobiología y Usos Médicos. Elementos. 2005;60:3 -9.

24. Hosking R D, Zajicek J P. Therapeutic Potential of Cannabis in Pain Medicine. British Journal of Anesthesia. 2008;101:59-68.

25. Durán M, Capellá D. Cannabis y Cannabinoides en el Tratamiento del Dolor Neuropático. Dolor. 2005;20:1

26. Pertwee R. Cannabinoids and Multiple Sclerosis. Molecular Neurobiology. 2007;36:45-59.

27. Zajicek J, Sanders H. Cannabinoids in Multiple Sclerosis (CAMS) Study: Safety and Efficacy Data for 12 Months Follow Up. Journal of Neurology, Neurosurgery and Psychiatry. 2005;76:1664 - 1669.

28. Svendsen K, Jensen T, Bach F. Does the Cannabinoid Dronabinol reduce Central Pain in Multiple Sclerosis? Randomized Double Blind Placebo Controlled Crossover Trial. British Medical Journal. 2004;329:253.

29. Killestein J. Safety, Tolerability, and Efficacy of Orally Administered Cannabinoids in Multiple Sclerosis. Neurology. 2002;58:1

30. Rog D, Nurmikko J. Oromucosal $\Delta 9$ - Tetrahidrocannabinol/ cannabidiol for Neuropathic Pain, Associated with Multiple Sclerosis: An Uncontrolled, Open Label, 2 - Year Extension Trial. Clinical Therapeutics. 2007;29:1

31. Rog D, Nurmikko T. Randomized, Controlled Trial of Cannabis- based Medicine in Central Pain in Multiple Sclerosis. Neurology. 2005;65:812-819. 